\title{
La Serbie à la croisée des chemins : corridors énergétiques, privatisations et guerre du gaz
}

Serbia on confluence: energetic corridors, privatization and war of gas

Serbien, ein neuerdings gegründeter Staat sucht nach seinem Platz auf einem im Umbruch balkanischen Schachbrett

\section{Alexis Troude}

\section{(2) OpenEdition}

Édition électronique

URL : http://journals.openedition.org/rge/2928

DOI : $10.4000 /$ rge.2928

ISSN : 2108-6478

Éditeur

Association des géographes de l'Est

Édition imprimée

Date de publication : 31 octobre 2010

ISSN : 0035-3213

Référence électronique

Alexis Troude, «La Serbie à la croisée des chemins : corridors énergétiques, privatisations et guerre du gaz », Revue Géographique de l'Est [En ligne], vol. 50 / 1-2 | 2010, mis en ligne le 23 septembre 2011,

consulté le 08 septembre 2020. URL : http://journals.openedition.org/rge/2928 ; DOI : https://doi.org/ $10.4000 /$ rge. 2928

Ce document a été généré automatiquement le 8 septembre 2020.

Tous droits réservés 


\title{
La Serbie à la croisée des chemins : corridors énergétiques, privatisations et guerre du gaz
}

\author{
Serbia on confluence: energetic corridors, privatization and war of gas \\ Serbien, ein neuerdings gegründeter Staat sucht nach seinem Platz auf einem im \\ Umbruch balkanischen Schachbrett
}

Alexis Troude

\section{Introduction}

1 La Serbie, Etat récemment créé, cherche sa place sur un échiquier balkanique. Ce territoire de $88631 \mathrm{~km} 2$ et presque 10000000 d'habitants, placé sur les confins orientaux de l'Union européenne (UE), est en pleine recomposition.

2 Passage obligé sur l'axe Rhin/Main/Danube et nœud de communication central de l'Europe du sud-est, la Serbie est l'objet des convoitises des firmes privées et des organisations internationales. A la confluence du corridor paneuropéen BudapestSalonique et de l'oléoduc américain Burgas-Dürres, une âpre bataille est engagée : les fortes ressources minières de la Serbie et du Kosovo sont en jeu. Or depuis le milieu des années 2000 s'est ajoutée la guerre du gaz où la Serbie joue malgré elle un rôle primordial : le projet américain Nabucco est concurrencé par le gazoduc South Stream, à travers lequel la Russie fait un come back saisissant dans la région.

Vient s'ajouter à cette géopolitique de l'énergie un processus d'intégration euroatlantique qui divise les territoires de la Serbie. Obligé de se plier aux normes libérales, le gouvernement serbe actuel est lancé dans une course aux privatisations ; c'est à l'aune de ce processus de préemption par les firmes et les pouvoirs locaux du tissu économique qu'il faut envisager un probable émiettement territorial. La Voïvodine, grenier à blé de la Serbie contrôlant l'accès au Danube, est agitée par un fort mouvement autonomiste. La Bosnie est aussi en proie aux mouvements centrifuges. 
Dans ce contexte d'éclatement territorial, la diplomatie serbe ne jure que par l'UE, tout en menant une active diplomatie vers les puissances montantes de la région (Russie et Chine), alors que les firmes étatiques de ces pays s'implantent progressivement dans tous les secteurs économiques.

\section{Les corridors énergétiques : l'Europe du sud-est au cœur du Grand Echiquier}

\section{A. Les Balkans comme nouveau débouché pour les hydrocarbures de mer Caspienne}

Les Balkans sont devenus ces dernières années un enjeu stratégique majeur pour les compagnies gazières et pétrolières internationales. En effet, à cause de la multiplication d'actions terroristes en Turquie et surtout la guerre d'août 2008 en Géorgie, l'espace sud-est européen reprend de l'importance dans la problématique de l'acheminement des énormes ressources en gaz et en pétrole, de la zone Caspienne-Asie centrale, vers le marché européen.

5 A la fin des années 1990, le premier projet de tube énergétique entre mer Caspienne et Europe a été le Bakou-Tbilissi-Ceyhan (BTC). Le BTC, ouvert en 2005, transporte le pétrole brut du champ pétrolifère d' Azeri-Chirag, en mer Caspienne, jusqu'à la mer Méditerranée. Avec $1776 \mathrm{~km}$, c'est le deuxième plus long oléoduc du monde après l'oléoduc Droujba qui relie l'Europe centrale à la Russie. L'oléoduc BTC, projet dirigé par un consortium d'entreprises occidentales chapeauté par la British petroleum (B.P.), a été conçu comme un tracé d'évitement de la Russie ; en effet, le BTC relie les champs pétroliers de la Caspienne au port turc de Ceyhan, en Méditerranée, en passant par l'Azerbaïdjan et le sud de la Géorgie. Le BTC a été en butte depuis sa mise en fonctionnement en 2006 à trois problèmes majeurs : des attentats de séparatistes kurdes dans le sud-est de la Turquie, la transgression des frontières de l'Arménie, au Nagorny-Karabagh, et le passage du BTC par l'Ossétie du sud, province de la Géorgie sécessionniste depuis 1992. La B.P. s'est vue obligée, dans ce contexte, de fermer en août 2010 le pipeline en raison d'une explosion sur son tronçon turc ; sa réouverture, initialement prévue en septembre 2010, en a été retardée.

L'autre projet occidental de contournement de la Russie est né un peu plus tard, mais a fonctionné les mêmes années que le BTC. Ainsi, l'oléoduc Bakou-Tbilissi-Batoumi était prévu pour déverser directement le pétrole de la Caspienne en mer Noire, pour ensuite être acheminé directement par bateau en mer Méditerranée, en passant par les Détroits. La Géorgie se trouvait au centre de ce dispositif, à la fois comme axe terrestre et comme débouché maritime. Or, la guerre d'août 2008 entre Russie et Géorgie a brusquement stoppé cette voie d'acheminement. Ainsi, la B.P. s'est trouvée obligée de fermer le 12 août l'oléoduc Western Route Export Pipeline et le gazoduc South Caucasus Pipeline en raison des événements survenus en Géorgie. Le 13 août, Robert Johnson, directeur du cabinet Eurasia Group, déclarait : La réputation de la Géorgie comme route alternative sécurisée a été compromise ...Si vous supprimez l'option géorgienne de la table... ça fait le jeu de la Russie, car la plupart des autres options viables passent par le territoire russe».

7 C'est là que la Russie a su se replacer dans ce jeu d'échec énergétique. Après les deux guerres de Tchétchénie de 1994 et de 1999, le président Poutine met en place un 
oléoduc et un gazoduc contournant la République sécessionniste : depuis 2000, ils relient directement Tenguiz au port russe de Novorossiisk, sur la mer Noire. Depuis le mois de novembre 1997, la capacité annuelle de transport du gazoduc TenguizNovorossiisk est de 17 millions de tonnes, sa longueur de $1411 \mathrm{~km}$. Le tracé russe est désormais à la fois une voie d'acheminement sécurisée des hydrocarbures et le seul réseau de tubes fonctionnant en continu entre mer Caspienne et mer Noire. Dans cette concurrence entrele consortium sous direction américaine "Albanian Macedonian Bulgarian Oil Corporation» (AMBO) et la compagnie d'état russe Lukoil, deux tracés sont opposés en Europe du sud-est :

- le tracé AMBO réunit les trois mers : Mer Caspienne (Bakou) / Mer Noire (Burgas) / Mer Adriatique (Dürres)

- le tracé Lukoil emprunte dans le Caucase la route Bakou - Tchétchénie - Novorossisk, puis de l'autre côté de la mer Noire l'axe orthodoxe Burgas (Bulgarie) - Alexandropouli (Grèce).

\section{B. La guerre du gaz dans les Balkans: Gazprom en train de gagner ?}

Dans cette bataille économique à échelle intercontinentale, le gaz est très vite devenu un enjeu majeur. Dans ce contexte, un consortium de compagnies gazières nationales européennes a été créé en 2004 sous l'égide de l'autrichien O.M.V., et il comprend quatre compagnies nationales de pays balkaniques (Hongrie, Roumanie, Bulgarie et Turquie), plus l'allemand R.W.E. et le français GDF-Suez. Ce consortium décidait de construire un gazoduc reliant les gisements d'Iran à l'Europe centrale en passant par le Caucase, avec un pays-pivot, la Turquie : c'est le projet Nabucco. D'une longueur de $3300 \mathrm{~km}$ et d'un coût prévu à 7,9 milliards d'euros, le gazoduc Nabucco devrait normalement fournir, à partir de 2014, 31 milliards de $\mathrm{m} 3$ de gaz par an aux pays d'Europe occidentale. Nabucco est soutenu par l'Union européenne (U.E.). Il a été conçu à la fois pour diversifier les sources d'approvisionnement énergétique de l'Europe, notamment d'un pays comme la Hongrie, qui dépend à $80 \%$ du gaz russe, et pour tenter de mieux contrôler l'approvisionnement du gaz venant de Russie, en cherchant d'autres sources d'approvisionnement.

Dans cette région du Moyen-Orient, Nabucco se trouve donc en concurrence avec le projet russe South Stream, qui utilise lui aussi à partir de la mer Caspienne le tracé du Caucase et le territoire de la Turquie. Or, cette concurrence plus la dépression économique en Europe font que les clients de ce gazoduc ne sont toujours pas identifiés ; l'appel d'offres auprès des clients aurait dû se dérouler entre juillet et octobre, pour une décision en décembre 2010. En octobre 2010, l'un des actionnaires repoussait le projet d'un an, en déclarant qu'aucune décision ne sera prise avant 2011. Avec du gaz naturel abondant dans différentes régions du globe, et des difficultés économiques en Europe de l'Ouest, l'avenir de ce projet reste donc incertain.

10 C'est pourquoi le projet South Stream, qui devrait normalement être achevé en 2015, est désormais un prétendant sérieux à l'acheminement des énormes ressources en gaz de la Russie, de l'Iran et du Turkménistan. Empruntant le tracé Burgas - Belgrade Zagreb pour ensuite se déverser à Trieste ou Vienne, le gazoduc South Stream est en concurrence directe dans les Balkans avec Nabucco, lequel utilisera le tracé Bakou Ankara - Sofia -Budapest - Vienne.

11 Dans un accord signéle 23 juin 2007 par Paulo Scaroni, président de la compagnie italienne E.N.I. et Alexander Medvedev, vice-président de Gazprom, le consortium 
international « South Stream AG », d'une valeur d'ensemble de 5,4 milliards de dollars, était créé.Dans ce consortium sont entrés à l'heure actuelle le français E.D.F., l'autrichien O.M.V., le turc Botas, le hongrois M.O.L. et le bulgare Bulgargaz. EDF a réussi à négocier une prise de participation de $10 \%$ dans le capital du consortium, contre des accords à long terme sur la livraison de gaz à plusieurs centrales thermiques le long du tracé. Entre 2011 et 2014, il est prévu de construire un gazoduc sous-marin de 900 kilomètres en mer Noire, au large des côtes turques, pour un investissement de 20 milliards de dollars. Cela permettrait à partir de 2014 de faire transiter par l'Europe du sud-est, afin d'approvisionner l'Europe de l'ouest, pas moins de 63 milliards de mètres cube de gaz par an.

Figure 1 : La bataille des « tubes » pour le marché européen du gaz

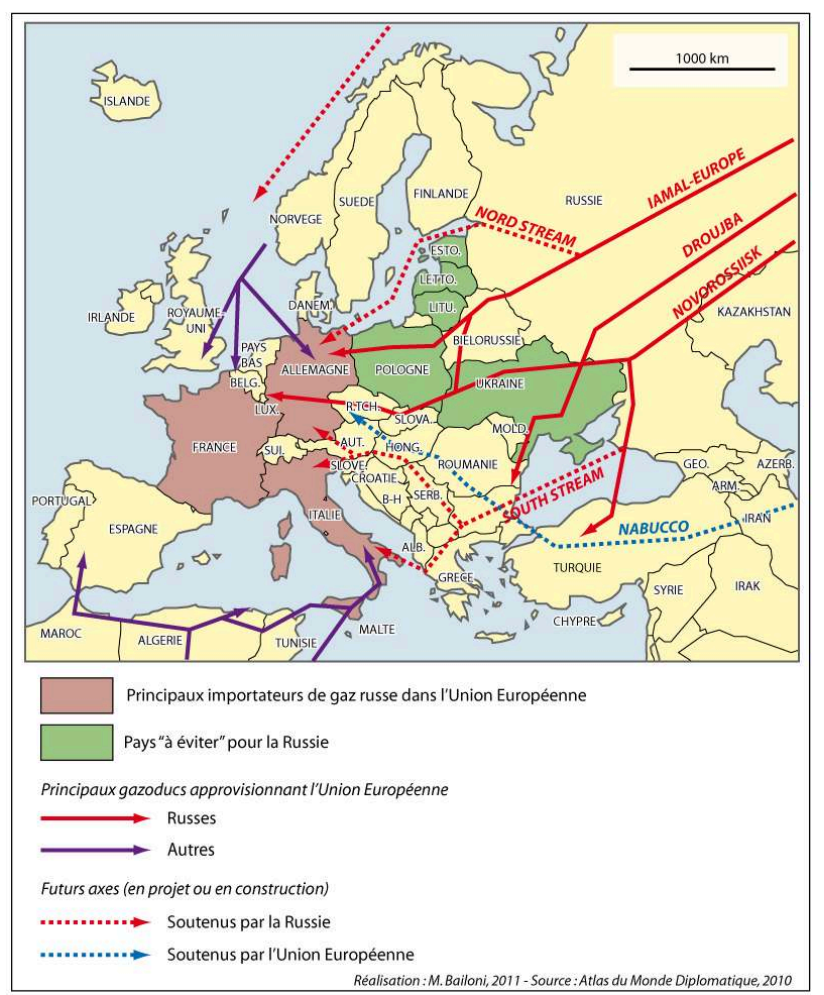

Dans ce dispositif de Gazprom dans les Balkans, la Serbie devient un élément central.

Le tracé à travers la Serbie sera de 450 kilomètres, soit le plus long de toute l'Europe du sud-est. De la frontière bulgare à l'est à la frontière hongroise au nord de la Serbie, le gazoduc passera par les villes de Nis et Belgrade avant de rejoindre l'espace de l'U... Y seront distribués 40 milliards de mètres cubes de gaz par an, avec des jonctions capitales pour plusieurs Etats de la région, selon deux raccordements : le premier vers le Kosovo et la Macédoine, l'autre en direction du Monténégro et de la Republika Srpska. L'enjeu est ici stratégique car le Kosovo dépend déjà à $80 \%$ pour son électricité de la Serbie et le raccord russo-serbe à cette fourniture de gaz accroîtra encore la dépendance géoéconomique du Kosovo vis-à-vis de la Serbie. Par ailleurs, des nations récentes comme la Macédoine et le Monténégro se voient de facto englobées dans un ensemble énergétique sous le giron de la Serbie.

A une plus large échelle se dessinent des recompositions intéressantes sur le plan des puissances énergétiques en Europe. Grâce à ce projet South Stream, une fois le tracé 
terminé, l'importance énergétique de la Serbie sera aussi grande pour l'U.E. que l'Ukraine aujourd'hui, alors que l'importance de l'Ukraine pour Moscou diminuerait. La Serbie dépend du soutien de Moscou pour le Kosovo, elle est donc pour la Russie un partenaire bien plus fiable que l'Ukraine qui, elle, fait le grand écart entre Russie et Etats-Unis.

\section{La stratégie de la Russie en Europe du sud-est}

\section{A. Conquêtes entrepreneuriales sur tout l'espace balkanique}

l'intérêt des entreprises russes pour ce jeune pays des Balkans (il est né en 2006) s'est manifesté tardivement, les capitalistes russes ont fait montre en quelques années d'une opiniâtreté impressionnante. Dans l'énergie, le géant russe du pétrole Lukoil a absorbé la société nationale serbe "Jugopetrol» et a remporté l'appel d'offres du gouvernement serbe concernant la privatisation de $79 \%$ des actions de la société de distribution de carburants «Beopetrol ». De son côté, la branche pétrolière de Gazprom "Gazpromneft» a absorbé en décembre 2008 «Naftna Industrija Srbije »(N.I.S.), principale compagnie pétrolière serbe, qui assurait jusque là la moitié de la distribution de l'or noir en Serbie. Gazpromneft a réalisé une assez bonne opération : pour 400 millions d'euros, soit moins que ce qu'offraient les autres prétendants comme l'autrichien O.M.V. ou le Hongrois M.O.L., la société russe possède désormais $51 \% \mathrm{du}$ capital de N.I.S. En contrepartie, Gazpromneft s'est engagée à investir dans la modernisation des raffineries serbes et dans la construction de centres de stockage de gaz naturel.

Par ailleurs, l'économie du Monténégro a été en l'espace de cinq ans majoritairement mise sous contrôle des prédateurs russes. La privatisation de la plus importante usine d'aluminium de l'ex-Yougoslavie, le Combinat d'aluminium de Podgorica (K.A.P.), a débouché sur son rachat en 2005, par le premier fabricant russe d'aluminium, « Rusal », contrôlé par Oleg Deripaska. Le groupe d'Oleg Deripaska, qui serait proche de Vladimir Poutine, a racheté les mines de bauxite de Niksic ainsi que le combinat d'aluminium de Podgorica. En 2006, il a remporté l'appel d'offres pour la privatisation des mines de charbon et de la centrale thermique de Pljevlja. C'est ainsi qu'il contrôlerait en 2010 plus de la moitié du PIB du Monténégro. De son côté, le géant russe Lukoil a racheté la firme "Jugopetrol Kotor".

De son côté, le géant russe du pétrole Lukoil a racheté la plus grande raffinerie en Bulgarie, Naftokihim à Burgas; la société russe a désormais le quasi-monopole sur le raffinage du pétrole en Bulgarie. Dans le nucléaire, des accords sur le combustible ont été passés avec la Bulgarie. La société russe " Rosatom » possède deux centrales dans le pays : Kozlodouy fonctionne déjà avec du combustible nucléaire russe et la société russe «Atomstroyexport » construira une deuxième centrale nucléaire dans la petite ville de Béléné. C'est un projet d'envergure, représentant un investissement de 4 milliards d'euros, avec la sous-traitance du français Areva, lequel a promis la réalisation définitive pour fin 2013. Comme en Serbie avec N.I.S., une polémique s'est engagée sur le manque de transparence de l'offre publique d'achat faite par la société russe en 2008. Mais après quelques atermoiements, le nouveau gouvernement bulgare s'est dit prêt à poursuivre sa collaboration. Là aussi, le facteur politique double les intérêts 
économiques car la Bulgarie doit défendre une position, difficile à tenir, entre bases américaines et tracé Nabucco d'un côté et une forte politique énergétique russe d'autre part.

18 La politique énergétique russe a aussi une forte emprise sur les pays à la jonction entre Europe centrale et Balkans que sont la Croatie, la Bosnie, la Hongrie et l'Autriche. On peut ainsi constater que Lukoil est actionnaire de l'entreprise hongroise M.O.L., et par ce biais de l'I.N.A. croate. C'est par ce moyen que Lukoil a pénétré sur le marché de la Bosnie-Herzégovine, s'assurant la majorité des actions de la firme bosniaque « Energopetrol »; l'entreprise russe « Neftgazinkor » a récemment acheté $65 \%$ des parts de deux raffineries en Republika Srpska.

19 La Croatie, quant à elle, consomme près de 3,2 milliards de mètres cubes de gaz par an, dont $60 \%$ proviennent de ses propres ressources et $40 \%$ sont importés de Russie. La tournée fin 2009 de l'envoyé de Vladimir Poutine, chargé de l'économie, à Belgrade et à Zagreb, est tout aussi parlante. Selon la presse croate, le représentant russe n'a pas seulement insisté pour que la Croatie accepte le projet d'oléoduc Druzba-Adrija, qui transformerait l'île de Krk, important port d'importation, en un port d'exportation du pétrole russe vers l'Europe occidentale ; il a également déclaré que la société Gazprom était prête à racheter la part du hongrois M.O.L dans l'industrie pétrolière croate I.N.A. Le 2 mars 2010, le premier ministre russe Vladimir Poutine se rendait en Croatie pour signer un accord gazier remplaçant l'actuel ; le but, selon l'homologue croate de Poutine, était clairement que "L'accord avec Gazprom expirant en fin 2010, la Croatie tient à obtenir des fournitures plus importantes de gaz".

$20 \mathrm{Au}$ bout du réseau South Stream mis en place par Gazprom, l'Autriche n'a pas été oubliée par les stratèges russes. Avec la prise de contrôle par Gazprom du terminal gazier de Baumgarten, la Russie maîtrise une véritable plaque tournante de distribution du gaz russe à l'ouest de l'Europe, par l'intermédiaire de l'Italie. Ce débouché de South Stream au cœur de l'Europe permettra aux Russes d'avoir un accès direct au marché gazier d'Europe occidentale à partir de 2015 : en doublant le réseau d'oléoducs "Bratsvo" desservant l'Allemagne et la Pologne, le gouvernement russe s'assure une voie alternative en cas de nouvelles "discussions" sur le transit en Ukraine ou en Biélorussie.

\section{B. La Serbie dans la tourmente : le rachat des compagnies de gaz par les Russes}

21 Le 22 janvier 2008 à Belgrade, après plusieurs mois de discussions bilatérales serborusses, le géant russe Gazprom rachetait la principale compagnie de distribution de gaz en Serbie, « Naftna Industrija Srbije » (N.I.S.). En vertu de cet accord, Gazprom versait en numéraire400 millions d'euros pour une prise de participation de 51\%; 500 millions étaient aussi prévus à moyenne échéance en investissement direct. Gazprom s'engageait aussi à plusieurs actions à une échéance très courte :

- construction de la plus grande centrale électrique de Serbie.

- installations souterraines de stockage d'une capacité de 3 milliards de m3 de gaz.

- construction, à Banatski dvor près de la frontière hongroise, d'un réservoir souterrain capable de contenir environ 300 millions de m3 ; de quoi fournir tous les pays d'Europe de l'ouest pendant une certaine période en cas de coupure du réseau.

- chiffre d'affaire stable pendant 10 ans sur le transit de gaz : 200 millions d'euros par an. 
Le 15 mai 2009, Dusan Bajatovic, le président de Srbijagas, signait avec Gazprom l'accord de création d'une filiale qui gèrera le projet Gazprom en Serbie : $51 \%$ des parts pour Gazprom, 49 \% pour Srbijagaz, avec un gazoduc qui ferait transiter jusqu'à 23,1 millions de $\mathrm{m} 3$ de gaz par an.

Les avantages énergétiques et économiques pour toute l'Europe du sud-est sont importants: d'une capacité de 63 milliards de $\mathrm{m} 3 / \mathrm{an}$, South Stream nécessitera un investissement d'une valeur de 20 milliards d'euros. Mais comme l'a dit lui-même Vladimir Poutine en 2008, mieux vaut un investissement lourd mais garantissant un transit sûr à travers des pays alliés, plutôt que de perdre 2 milliards à cause de la controverse avec le gouvernement ukrainien de l'époque. En outre, l'apport pour les économies des Balkans touchées durement par la crise n'est pas négligeable, puisque South Stream représente la création de 100.000 emplois d'ici 2015, pendant la durée de réalisation du gazoduc : cette série d'accords entre Russie et Serbie permettent à cette dernière d'avoir une véritable relance économique. Plus important encore, la Serbie deviendrait, au cœur du dispositif South Stream, un centre de distribution de gaz pour toute l'Europe du sud-est : 30 milliards m3 de gaz/an à destination de la Roumanie, la Hongrie, la République tchèque, l'Italie et l'Autriche.

Tableau : Arguments pour et contre le rachat du N.I.S. par Gazprom

\begin{tabular}{|c|c|}
\hline $\begin{array}{c}\text { ARGUMENTS EN FAVEUR DU RACHAT DE N.I.S. PAR } \\
\text { GAZPROM } \\
\end{array}$ & $\begin{array}{c}\text { ARGUMENTS CONTRE LE RACHAT DE N.I.S. PAR } \\
\text { GAZPROM }\end{array}$ \\
\hline $\begin{array}{l}\text { Sécurité énergétique (énergie assurée pour } 50 \text { ans) : garantie } \\
\text { d'approvisionnements par le ler producteur mondial vis-à-vis } \\
\text { d'une puissance régionale }\end{array}$ & $\begin{array}{l}\text { Société nationale de distribution de gaz bradée : actions placées en } \\
\text { dessous du cours du marché }\end{array}$ \\
\hline $\begin{array}{l}\text { Coûts garantis pendant } 25 \text { années à un niveau bien en dessous du } \\
\text { cours mondial }\end{array}$ & $\begin{array}{l}\text { Principe de la concurrence non respecté : } \\
\text { aucune offre publique d'achat } \\
\text { (prix fixé de façon unilatérale) }\end{array}$ \\
\hline $\begin{array}{l}\text { Construction d'installations (gazoducs, terminaux, centres de } \\
\text { stockage) } \\
\text { Effet d'entrainement sur le commerce (investissements) }\end{array}$ & $\begin{array}{l}\text { Désaisissement du principe de souveraineté économique au profit } \\
\text { d'un groupe étranger }\end{array}$ \\
\hline $\begin{array}{l}\text { Compensation économique au soutien politique de la Russie pour } \\
\text { le maintien de la province du Kosovo-Métochie au sein de la } \\
\text { Serbie }\end{array}$ & $\begin{array}{l}\text { Balkans deviennent espace russe: droits de concession passés sous } \\
\text { le contrôle de Moscou }\end{array}$ \\
\hline Courants Slavophiles à Belgrade (Radicaux, Kostunica) & $\begin{array}{l}\text { Courants occidentalistes } \\
\text { (gouvernement et libéraux du Parti démocrate) }\end{array}$ \\
\hline
\end{tabular}

Tout de suite, une polémique éclatait en Serbie, la partie libérale du gouvernement hurlant aux loups en dénonçant un accord trop vite signé, sans respect des règles de transparence inhérentes à ce genre d'opérations. Le 31juillet 2009, le Ministre des Finances, Mladen Dinkic, proclamait : « Nous avons subi une humiliation en acceptant l'offre de Gazprom ». En fait, les libéraux, majoritaires au gouvernement serbe, soutiennent que le principe de la concurrence a été violé et que la société NIS a été bradée avec un montant d'achat dérisoire. A plus longue échéance, les libéraux estiment que la Russie maitrise désormais une trop grande partie de la filière énergétique de la Serbie (distribution-stockage du gaz-raffinerie de pétrole) et que l'accord n'est pas suffisamment clair quand à l'assurance de revenus à moyen terme.

Les partisans de l'accord soutiennent, dans une perspective nationale, que cet accord gazier russo-serbe est non seulement une garantie énergétique à moyenne échéance, mais surtout redonne une impulsion à une économie serbe moribonde. A plus long terme, l'accord garantira, selon ses défenseurs, un lien stratégique indéfectible avec le plus grand producteur mondial d'hydrocarbures, à un moment où l'U.E. hésite à s'engager davantage dans l'intégration économique et politique de la Serbie. Mais derrière ces arguments économiques, une réalité géographique place en première 
position les partisans de l'accord avec Gazprom. Dans la question capitale d'une jonction éventuelle entre "South Stream" et "Nabucco", Dusan Bajatovic, Directeur général de "Srbijagaz" est on ne peut plus clair : « Notre compagnie ne participera pas aux négociations sur la jonction des deux gazoducs, mais alors que le projet Nabucco n'en est qu'à ses balbutiements, le projet South Stream est une réalité tangible ».

\section{Processus d'éclatement territorial lié aux enjeux des hydrocarbures}

Toutes ces luttes d'influence exercent une forte pression économique et politique sur les espaces d'Europe du sud-est. La recherche de nouveaux axes de pénétration énergétiques, à travers des territoires souvent accidentés, explique les processus actuels de mouvements centrifuges. C'est le cas notamment en Serbie et sur ses marges, car ce pays situé à la confluence des tubes énergétiques Est-Ouest dans les Balkans, est en proie aux irrédentismes. L'espoir de développement économique ouvre de formidables perspectives d'essor grâce à l'or noir. Voici trois exemples de saillants, au cœur de l'hinterland balkanique, indispensables pour le transit d'hydrocarbures et qui provoquent de fortes tensions séparatistes.

\section{A. Le Kosovo à l'intersection des corridors énergétiques}

A l'intersection des corridors paneuropéens IV, VIII et X définis à la conférence d'Helsinki en 1997, le Kosovo est au cœur d'un réseau de première importance. Véritable entonnoir lorsqu'on observe le Kosovo du point de vue centre-européen, la province s' "ouvre" au corridor VIII à travers sa façade tournée au Sud vers la Macédoine. Lorsqu'on sait que le Kosovo regorge de minerais rares comme le tungstène ou le zircon utile aux têtes de missiles, on comprend mieux l'intérêt porté par les EtatsUnis à l'axe Burgas/Dürres. A l'Est, dans la basse-Morava, le corridor $\mathbf{X}$ draine déjà entre Budapest et Salonique des flux commerciaux vitaux pour la Macédoine ou la Serbie. A l'ouest, le corridor IV Bucarest-Trieste aiguise les appétits des Russes. C'est pourquoi les projets de tuyaux gaziers et pétroliers soutenus par Moscou contrecarrent au Kosovo les projets américains AMBO (oléoduc) et Nabucco (gazoduc) de faire transiter par les Balkans les hydrocarbures en provenance d'Asie centrale. Le Kosovo est aussi convoité par les grandes puissances économiques pour ses importantes richesses minérales : $4 \mathrm{e}$ réserve mondiale de lignite, plomb, zinc et cuivre aux teneurs exceptionnelles.

Figure 2 : Le Corridor 10 


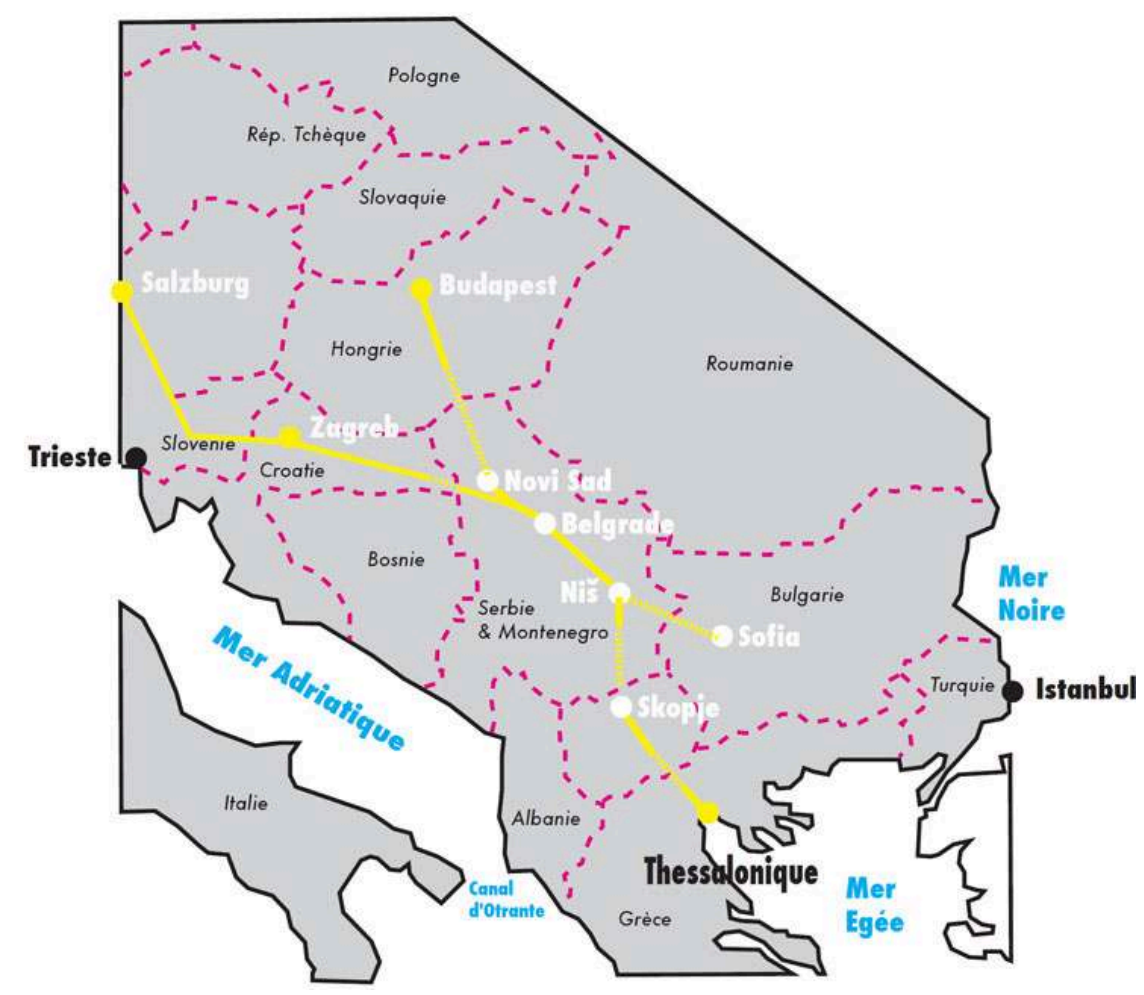
Balkans se trouve dans le Sud-Est du Kosovo, c'est-à-dire à 35 kilomètres de l'intersection des corridors VIII et X. Construite à partir de 1999, elle couvre 750 hectares, et peut abriter jusqu'à 7000 soldats. Avec Bondsteel, c'est une présence à long terme des Etats-Unis dans les Balkans qui s'esquisse. Bondsteel est une plate-forme de départ pour une future intervention militaire des Etats-Unis dans la région. En cas de crise en Macédoine ou au Monténégro voisins, les forces américaines pourraient intervenir plus rapidement. En janvier 1999, l'éditorialiste du "Washington Post» déclarait sans ambages que «Le Moyen-Orient devenant de plus en plus fragile, nous allons avoir besoin de bases et de droits de survol aérien dans les Balkans pour protéger le pétrole de la mer Caspienne ».

\section{B. Bosnie-Herzégovine: le corridor de Brcko, plate-forme énergétique Serbie/Bosnie/Croatie}

Les Accords de Dayton, signés en 1995, ont créé en Bosnie-Herzégovine deux entités distinctes : la "Fédération croato-musulmane » et la «Republika Srpska ». Dans cet aménagement administratif confédéral, un statut particulier a été attribué en 2002 au canton de Brcko, seul raccord entre Serbie et entité serbe de Bosnie : celui de corridor sous contrôle international.

31 L'intérêt stratégique de la ville de Brcko explique l'attention portée par la communauté internationale. Placé sur la rivière Sava, Brcko est actuellement le seul port de Bosnie sur cet affluent du Danube : Brcko est ainsi un lien direct avec la Croatie et la Slovénie à l'Ouest par la Sava, la Hongrie et la Roumanie à l'Est par le Danube. D'autre part, ce port sur la Save accueille traditionnellement le trafic remontant des affluents que sont la Bosna et la Drina. Le gouvernement italien etl' «International Management Group ", agence de l'UNHCR, ont depuis 2001 investi dans la

Revue Géographique de l'Est, vol. 50 / 1-2 | 2010 
reconstruction du port et la Croatie a dragué en amont sur la Sava afin d'approfondir son lit. Ainsi, le contrôle du carrefour fluvial qu'est Brcko permettra bientôt à la Bosnie d'avoir une ouverture vers le Danube qui, même sans équilibrer la perte d'un débouché sur l'Adriatique, est potentiellement intéressant.

Or Brcko se trouve à 32 kilomètres au sud du futur tracé du South Stream, sur le raccord que le gouvernement serbe, en entente avec la Russie, a prévu de construire pour fournir de l'énergie gazière à la Republika Srpska. L'enjeu est ici de taille pour le gouvernement de Serbie : approvisionner les populations serbes en livraisons durables de gaz de l'autre côte de la frontière, c'est-à-dire en Bosnie. Mais il y a aussi des enjeux politiques :

- pour Belgrade, s'assurer de la fidélité du dirigeant des Serbes de Bosnie, Milorad Dodik, qui jusque là menait une politique assez indépendante.

- pour Sarajevo, les disputes autour du corridor de Brcko lui donnent un moyen de pression sur Belgrade.

On comprend donc mieux l'action menée par la communauté internationale sur ce bout de territoire étroit mais stratégique.

\section{En Serbie : la Voïvodine sur le tracé South Stream et Nabucco}

La Voïvodine est une des deux Provinces Autonomes de la Serbie. Peuplée de 2.031.000 habitants et contrôlant tout le cours du Danube en Serbie, la Voïvodine est une riche région agricole, qui fournit un tiers du blé du pays. Bien que les Serbes y soient maintenant majoritaires (65\%), elle compte une forte minorité hongroise $(290.000$ habitants) et 26 minorités ethniques.

Or la Voïvodine occupe une position stratégique entre l'Europe centrale et les Balkans, passage obligé vers la Hongrie et la Roumanie. Traversée du Nord-Ouest au Sud-Est par le Danube, la Voïvodine fait donc partie du système de canaux à grand gabarit Rhin/ Main/Danube. Les couloirs européens IV (Berlin-Mer Noire) et X (Autriche-Mer Egée) la traversent, ainsi que les tracés des futurs gazoducs "South Stream » et "Nabucco ». C'est pourquoi les grands industriels autrichiens, hongrois et russes tentent de s'implanter en Voïvodine. Les compagnies autrichienne O.M.V. et hongroise M.O.L. ont manifesté leur désir de racheter la compagnie pétrolière serbe N.I.S. ; O.M.V. s'était intéressée dès 2006 à l'acquisition d'une raffinerie à Novi Sad. Mais les Russes ont été plus rapides et Gazprom a acquis 51 \% de N.I.S. en décembre 2008. La Voïvodine est ainsi devenue le terrain d'une confrontation stratégique entre une Russie misant sur la Voïvodine comme couloir énergétique vers l'Europe et de contournement de l' « ennemi » ukrainien ». Ce n'est pas par hasard que Gazprom a décidé en janvier 2008 que le principal centre de stockage gazier russe dans les Balkans serait établi dans le futur en Voïvodine.

En octobre 2008, le Parlement de Novi Sad a examiné un projet de nouveau statut lui conférant tous les attributs d'un Etat (gouvernement, banque centrale et représentations à l'étranger). Mais derrière cette question constitutionnelle se cache la question du séparatisme hongrois. Dans un document de la "Coalition hongroise ", son dirigeant Istvan Pastor déclarait explicitement: «Le but de la coalition hongroise est la consolidation significative de l'autonomie de la Voïvodine à travers la modification de la Constitution de la République de Serbie, pour la reconstruction dans notre Province du législatif, de l'exécutif et, en partie, du judiciaire». Ainsi, la Hongrie pourrait reconnaître la 
Voïvodine comme un Etat, faisant dès lors de la Serbie une Fédération serbo-hongroise. En fait, la Voïvodine constitue aux yeux de ses dirigeants un bloc multinational au nord du Danube, sorte de zone-transitoire entre Serbie et Hongrie : elle aurait vocation à devenir une Eurorégion rattachée à l'Europe centrale, aux confins méridionaux de l'U.E.

\section{Conclusion}

La petite Serbie se trouve donc à un moment-clé de son histoire : soit elle intègre le giron de l'U.E. et oriente son économie sur un axe nord-sud, soit elle s'arrime à un bloc oriental guidé par la Russie.

Dans la perspective d'une intégration accélérée à l'espace euro-atlantique, la Serbie deviendrait à nouveau, comme elle l'a été au début du $\mathrm{XX}^{\circ}$ siècle, la nation centrale des alliances occidentales dans les Balkans ; cela lui permettrait de rentrer de plain-pied dans la mondialisation, en devenant le pivot des échanges intra et extra européens sur les marges de l'UE. Cela explique que le camp libéral en Serbie soit aussi accroché à l'axe UE/USA. En relevant les valeurs européennes de la Serbie, ils veulent à tout prix s'arrimer à un des pôles majeurs de la Triade, même si cela passe par l'abandon de la souveraineté nationale et par l'émiettement territorial.

Par contre, si la pénétration économique de la Russie continue à ce rythme, il est à craindre que la Serbie ne se transforme en point d'accroche avec l'Eurasie. En effet, il est incontestable que face à ces menaces centrifuges (Kosovo, Voïvodine) et à la crise économique durable, un mouvement d'opinion s'est répandu en faveur de la recherche d'un allié sûr et économiquement solide: c'est la Russie qui joue ce rôle, comme cela pourrait à l'avenir être la Chine.

Il est intéressant de voir dans cette configuration le cheminement de la Bulgarie, nation traditionnellement russophile mais dans l'espace euro-atlantique. Des similitudes apparaissent avec la Serbie, comme les forts investissements russes et la russophilie du peuple bulgare. Mais à la différence de la Serbie, les pressions économiques n'ont pas débouché sur des mouvements centrifuges : la position plus centrale de la Serbie explique les visées territoriales à l'œuvre chez cette dernière.

1 Dans ce contexte, le combat entre Occidentaux et Russes pour l'acheminement du gaz d'Asie centrale vers l'Europe prend tout son sens. Gazprom a su par une habile politique fondée sur des investissements chez ses alliés étendre son réseau à tous les pays des Balkans, mais l'U.E. et les Etats-Unis n'ont pas dit leur dernier mot.

\section{BIBLIOGRAPHIE}

Foucher M., Fragments d'Europe, atlas de l'Europe médiane et orientale, Fayard, 1998.

Glamotchak M., « Russie-Serbie : l'âme slave à l'épreuve de l'énergie », Outre-Terre n 27, février 2011. 
Mityajev O., « La vente de N.I.S. à Gazprom », RIA Novosti, 23 janvier 2008, p 16.

Otasevic A., «Le rachat de Nis par Gazprom : enjeux et limites », Geopolitika n²5, octobre 2010, pp 33-38.

Ougartchinska R., La Guerre du gaz, Editions du Rocher, 2008.

Paniouchkine V., Gazprom, l'arme de la Russie, Actes Sud, 2008.

Petrovic J., « South Stream est une priorité pour la Serbie », Politika, 23 février 2010, p 31.

Troude A., 2008, «Le Kosovo, un quasi-état dans la nouvelle guerre froide », Diplomatie n³2, maijuin 2008, pp 56-61.

\section{RÉSUMÉS}

La Serbie, Etat récemment créé, cherche sa place sur un échiquier balkanique en pleine recomposition. Ce territoire de $88631 \mathrm{~km} 2$ et presque 10000000 d'habitants, placé sur les confins orientaux de l'Union européenne, connaît à la fois un processus d'intégration européenne et cherche sa place dans l'Europe du sud-est.

L'espace balkanique est devenu, depuis la découverte d'importants gisements d'hydrocarbures en mer Caspienne en 1997, une plaque tournante des réseaux de gazoducs et d'oléoducs entre Asie et Europe. A l'intersection des axes d'influence de l'UE, des Etats-Unis et de la Russie, une âpre bataille mêlant compagnies multinationales et institutions internationales est engagée, où les tracés de tubes énergétiques évoluent au gré des situations géopolitiques. Or depuis le milieu des années 2000 s'est ajoutée la guerre du gaz, où la Serbie joue malgré elle un rôle primordial : le projet américain Nabucco est concurrencé par le projet de gazoduc russe South stream.

A travers une politique énergétique agressive, la Russie fait un retour saisissant dans toute l'Europe du sud-est. Tout le long du tracé du South stream entre Mer noire et mer Adriatique, les entreprises russes investissent massivement dans les secteurs de l'énergie, des transports et du tourisme, très souvent avec l'aide indirecte de l'Etat. Cela permet à la Russie de prendre position sur tout l'espace balkanique, grâce à une influence économique dans des Etats retrouvéscomme la Bulgarie, la Bosnie-Herzégovine, la Croatie, le Monténégro et la Serbie. Dans ce dernier, le rachat du secteur gazier et pétrolier par les géants russes Gazprom etLukoil leur a permis de contrôler la distribution d'énergie dans un pays central de leur dispositif d'expansion économique en Europe du sud-est, créant une vive polémique dans les milieux politiques et économiques du pays.

C'est à l'aune de ce processus endogène de préemption par les firmes et les pouvoirs locaux du tissu économique qu'il faut envisager un probable émiettement territorial. Les autorités albanaises du Kosovo La diplomatie serbe ne jure que par l'UE, tout en menant une active diplomatie vers les puissances montantes de la région (Turquie, Russie et Chine), alors que les firmes étatiques de ces pays s'implantent progressivement dans tous les secteurs économiques. La diplomatie serbe ne jure que par l'UE, tout en menant une active diplomatie vers les puissances montantes de la région (Turquie, Russie et Chine), alors que les firmes étatiques de ces pays s'implantent progressivement dans tous les secteurs économiques.

Les régions à l'intersection des corridors pan-européens IV, VIII et X, ont auto-proclamé leur indépendance pour mieux contrôler le passage des hydrocarbures de mer Caspienne sur leur territoire. Dans le nord-est de la Bosnie-Herzégovine, le corridor de Brcko est un bon exemple de l'opposition de stratégies antagonistes pour le contrôle d'axes fluviaux et de tubes énergétiques primordiaux dans l'espace balkanique. La Voïvodine, grenier à blé de la Serbie et contrôlant l'accès au Danube, est agitée par un fort mouvement autonomiste : l'enjeu est ici de contrôler le 
trafic fluvial sur l'axe Rhin/Main/Danube. Dans ce contexte d'éclatement territorial, la place de la Serbie dans le système euro-atlantique est à la fois intégrationniste et centrifuge.

Serbia, a recently created State, is looking for its rank on the Balkans stage. This territory of 88 $631 \mathrm{~km} 2$ and almost 10000000 inhabitants, placed on the oriental borders of the European Union (EU), is in full reorganization.

An unavoidable passage on the axis the Rhine / Main/ Danube and a central knot of communication of the South-East Europe, Serbia arouses the greed of private firms and international organizations. In the confluence of the Paneuropean corridor Budapest-Salonique and the American oil pipeline Burgas-Dürres, a hard battle is being held: the strong mining resources of Serbia and Kosovo are at stake. Now since the midst of the 2000s the war of the gas is a new element in which Serbia, unintentionally, plays an essential part the American Nabucco project is competed by the gas South Stream pipe-line, through which Russia makes a striking come back in the region.

A process of euro-Atlantic integration is to be added to this geopolitics of the energy which is dividing the territories of Serbia. Compelled to comply with the liberal standards, the current Serbian government is engaged in a race to privatization; it is in the light of this process of preemption of the economic infrastructures by the firms and the local powers that it is necessary to envisage a possible territorial explosion. Voivodine, the wheat "belt" of Serbia, controlling the access to the Danube, is shaken by a strong separatist movement. Bosnia and Herzegovina is also in the grip of centrifugal movements. In this context of territorial explosion, the Serbian diplomacy has only eyes for the EU, leading an active diplomacy towards the rising powers of the region (Russia and China), while the state firms of these countries are getting gradually established in all the branches of industry.

Dieses Territorium $88631 \mathrm{~km} 2$ und fast 10 Millionen Einwohner, das auf den östlichen Grenzbereichen der EU liegt, erlebt einen europäischen Integrationsprozess und sucht nach seinem Platz in Südosteuropa.

Seit der Entdeckung wichtigster Erdöl- und Erdgasvorkommen in dem kaspischen Meer in 1997 ist der balkanische Raum eine Drehscheibe der Erdgas- und Erdölleitungsnetze in Asien und Europa geworden. An der Schnittstelle zwischen den Einflusssphären der EU, der Vereinigten Staaten und Rußlands eröffnet sich eine bittere Schlacht zwischen multinationalen Firmen und Institutionen, wo die Trassen der Energieleitungen nach den geopolitischen Lagen sich entwickeln. Seit der Mitte der 2000er Jahre hat sich der Erdgaskrieg hinzugefügt, in dem Serbien eine entscheidende Rolle spielt: das amerikanische Projekt Nabucco steht in Konkurrenz mit der russischen Erdgasleitung South Stream.

Durch eine aggressive Energiepolitik macht Rußland eine erstaunliche Wiederkehr im ganzen Südosteuropa. Entlang der Southstreamtrasse zwischen dem Schwarzen Meer und dem Adriatischen Meer investieren die russischen Firmen massiv in den Energie-, Verkehrs- und Fremdenverkehrssektoren, oft mit der inderikten Hilfe des Staaten. Dank des wirtschaftlichen Einflusses in wiedergefundenen Ländern wie Bulgarien, Bosnien, Kroatien, Montenegro und Serbien kann Rußland einen Platz auf dem balkanischen Raum nehmen. Dank des Einkaufs der Erdgas- und Erdölsektoren konnten die russischen Firmen Gasprom und Lukoil die Energieversorgung in Serbien kontrollieren, einem Land im Kern ihres wirtschaftlichen Entwicklungsplanes in Südosteuropa. Diese Entwicklung hat eine starke Polemik in den politischen und wirtschaftlichen Kreisen des Landes.

Eine territoriale Zersplitterung ist $\mathrm{zu}$ erwähnen zum Maßstab dieses endogenenVorkaufsprozesses der wirtschaftlichen Struktur durch die lokalen Firmen und Mächte. Die serbische Diplomatie schwört nur auf der EU, während sie eine aktive Diplomatie 
nach den aufsteigenden Mächten der Region (Türkei, Rußland und China) betreiben und die Firmen dieser Länder allmählich in allen wirtschaftlichen Sektoren sich ansiedeln.

Die albanischen Mächte in Kosovo haben ihre Abhängigkeit selbstproklamiert, um die Kohlenwasserstoffeleitungen aus dem Kaspischen Meer zu kontrollieren. Im Nordosten von Bosnien ist der Brcko Korridor ein gutes Beispiel für gegensätzliche Strategien, um wichtige Fluvialachsen und Energieleitungen im balkanischen Raum zu kontrolllieren. Voïvodien, der Kornspeicher Serbiens und der den Zugang zur Donau kontrolliert, ist von einer starken Autonomiebewegung gebrodelt: der Fluvialverkehr auf dem Main/Donau Achsen zu kontrollieren steht auf dem Spiel. In diesem Rahmen einer territorialen Zersplitterung hat Serbien in dem euro-atlantischen System einen Integrations- und Zentrifugalplatz.

INDEX

Schlüsselwörter : Energie Korridore, Industrie-Lobbying, strategische Allianzen, Transnational Mots-clés : alliances stratégiques, corridors énergétiques, lobbying industriel, transnationales Keywords : energetic corridors, industrial lobbying, strategic alliances, transnational

\section{AUTEUR}

\section{ALEXIS TROUDE}

Chercheur au Laboratoire Géo-cités - Université Paris I-Sorbonne - 12 place du Panthéon 75231 PARIS Cédex 05 / Chargé de cours à l'Université Versailles-Saint-Quentin troudealexis@yahoo.com 